\title{
The effect of composition and processing on electric characteristics of XLPE in HVDC cable applications
}

\author{
A. Fazal, M. Hao, A. S. Vaughan, G. Chen \\ Tony Davis High Voltage Laboratory \\ University of Southampton \\ Southampton, SO17 1BJ, UK \\ a.fazal@soton.ac.uk
}

\author{
J. Cao, H. Wang \\ Smart Grid Research Institute \\ Beijing, China
}

\begin{abstract}
Polyethylene exhibits many key characteristics including low dielectric loss, high breakdown strength and good processability. Most modern extruded high voltage cables employ cross-linked polyethylene (XLPE) as the insulation material. The main advantage of XLPE is its excellent thermo-mechanical properties; it is relatively cheap and has low dielectric loss and low conductivity making it an ideal material for this application. Crosslinking enhances a number of thermo-mechanical properties such as deformation resistance at higher temperatures, tensile strength and creep properties. In comparison with low density polyethylene (LDPE), the heat deformation characteristics of XLPE are superior and, for this reason, XLPE is currently the most common insulation material for power cables ranging from low to high voltages. This paper reports on an investigation into the development of a new XLPE formulation for use in high voltage direct current (HVDC) cable applications. Specifically, the electrical performance of two novel LDPE resins are compared with an industrial standard (reference) LDPE material. For crosslinking, dicumyl peroxide (DCP) was selected, as the decomposition temperature is high enough to prevent pre-curing during processing and to allow an efficient and rapid crosslinking at moderate temperatures. Moreover, the behavior of various systems is compared in terms of electrical breakdown performance and the influence of material composition and processing on these parameters is described.
\end{abstract}

Keywords - Crosslinking; HVDC cable; LDPE; XLPE

\section{INTRODUCTION}

In the modern world, cables play a vital role in the transmission and distribution of electrical power. The typical construction of power cables includes a conductor surrounded by insulation and semi-conductive (semicon) screens. The semicon provides a smooth interface between the insulation and the conducting parts, thus preventing the concentration of electrical stress within the insulation material and, thereby, reducing the likelihood of premature breakdown. Indeed, the desirable electrical properties of LDPE were identified at an early stage and this type of material has now been used as electrical insulation for over 60 years. Today, however, thermoplastic LDPE has been replaced by XLPE and it is the most common insulation material for power cables designed for high voltages [1]. Crosslinking introduces a network in which the polymer structure is modified through the formation of chemical bonds between adjacent polymer chains [1].
To date, three crosslinking methods have been commercially applied, these are peroxide, silane and radiation [2]. Introducing crosslinking into the polyethylene can be achieved in several possible ways [3], but the most commonly used method in industrial settings today is peroxide crosslinking [4]. A number of peroxide crosslinking agents are suitable for LDPE, but dicumyl peroxide (DCP) is most frequently used in this context. The decomposition temperature of DCP is high enough to keep the pre-curing during processing at a low level, and sufficiently low to allow an efficient and rapid crosslinking at moderate temperatures, without unnecessary degradation [4]. Crosslinked polyethylene is generally used in cables to achieve enhanced thermomechanical stability at high temperatures $[3,5]$. The evolution of insulation materials has been driven by the need for higher reliability and lower cost. Due to ohmic losses, the cable conductor becomes heated at high power loads and this could lead to the melting temperature of the insulation material being approached. At such temperatures, there is a risk of the cable being deformed, such that the conductor might penetrate the insulation. This ultimately would result in electrical breakdown. However, using XLPE, it is possible to operate a cable with a conductor temperature of $90{ }^{\circ} \mathrm{C}$, which is $20^{\circ} \mathrm{C}$ higher than uncrosslinked polyethylene [1].

In this paper, an attempt is made to review commercial feasibility of XLPE material for the development of advanced XLPE-HVDC extruded cables. This mainly focuses on the methodologies for material characterization with respect to their influence on DC breakdown strength.

\section{EXPERIMENTAL}

\section{A. Materials}

Three different LDPE grade material $A$ (reference) supplied by ExxonMobil and $B$ and $C$ supplied by Smart Grid Research Institute are evaluated. The crosslinking agent, (DCP), was supplied by Sigma-Aldrich. LDPE is well known for its homogeneity and its good extrusion behavior, therefore, DCP is an ideal candidate, as the decomposition conditions is well above the LDPE melt temperature [1]. Moreover, this is an efficient way to achieve crosslinking as the radical reaction occurs at temperatures above the LDPE melting point, that is, $\sim 120^{\circ} \mathrm{C}$. 


\section{B. Sample preparation and crosslinking process}

Materials were processed in a Thermo PolyLab mixer for 15 min at $120^{\circ} \mathrm{C}$. The mixing chamber was filled with pellets along with $2 \%$ crosslinking agent and the evolution of torque and temperature were recorded. The different stages involved in the production process of the materials (before and after melt mixing) are shown in Fig. 1. Crosslinking of the material was performed in a Grasby Specac hydraulic press. The initial temperature to melt the material was set at $120^{\circ} \mathrm{C}$ for $2 \mathrm{~min}$ and then increased to $180^{\circ} \mathrm{C}$ for $5 \mathrm{~min}$ to activate crosslinking in the material. The applied load (5 tons) was maintained during the cooling process and the samples were removed from the hot press once $120^{\circ} \mathrm{C}$ was reached and further cooled at room temperature.
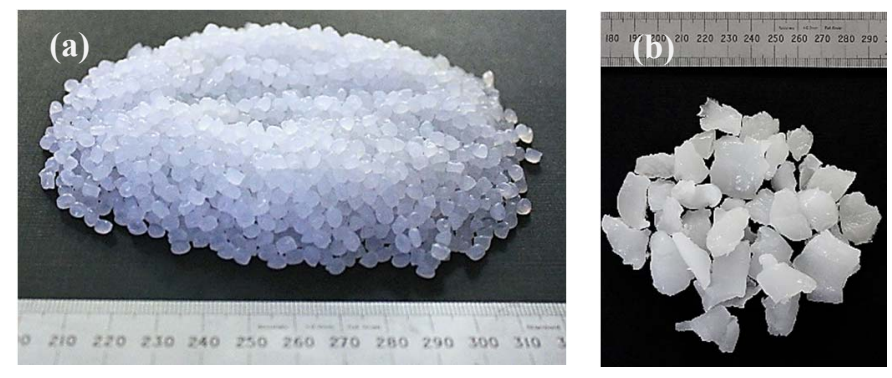

Fig. 1. Formation of the material during the mixing process. (a) LDPE pellets, (b) blended LDPE including DCP.

\section{Degassing}

In common with other crosslinking agents, DCP produces by-products (curing decomposition products) within the material as a result of the crosslinking reaction, which can influence the electrical properties of the materials $[6,7]$. Therefore, all XLPE samples were vacuum processed to remove volatile residual by-products, which may have originated from the crosslinking process. For this, samples were left in a vacuum oven for $6 \mathrm{~d}$ at a constant $70^{\circ} \mathrm{C}$. Electrical tests on undegassed samples were also performed.

\section{Analysis}

Differential scanning calorimetry (DSC) analysis of the LDPE base material mixed with the crosslinked agent (i.e. DCP) was carried out using a Perkin Elmer DSC 7. For DSC scan, a sample $15-20 \mathrm{mg}$ in mass was used and all scans were obtained at a heating rate of $10{ }^{\circ} \mathrm{C} / \mathrm{min}$ in an inert nitrogen atmosphere. The instrument was routinely calibrated using high purity indium.

Breakdown tests on all XLPE formulations, both degassed and undegassed, were performed. A standard electrical test procedure was followed, in which a thin film sample was immersed in Dow Corning silicone oil (200/20cs) between two $6.3 \mathrm{~mm}$ diameter steel ball-bearing electrodes. The voltage was raised from zero up to the level of breakdown at a rate of 100 $\mathrm{V} / \mathrm{s}$. In total, twenty tests on each batch (degassed and undegassed samples) were performed and the resulting normalized breakdown strength data (voltage divided by the sample thickness) were analyzed using Reliasoft Weibull 7++ software. To ensure the required voltage for breakdown was within the limitations of the electrical equipment, the samples thickness were kept at $75 \mu \mathrm{m} \pm 5 \mu \mathrm{m}$. Since samples thickness is known to affect the measured electrical strength, it was essential to maintained a uniform sample thickness [8].

\section{RESULtS}

\section{A. Material Processing}

For the material preparation, it was necessary to know the parameters for ideal mixing duration; mixer RPM (torque) and the required mixing temperature. Fig. 2 highlights variations in melt characteristics corresponding to various mixing conditions. It is worth noting that the increase in temperature $\sim 10^{\circ} \mathrm{C}$ from the set point temperature is caused by the internal friction generated within the material, i.e. the interaction of material and rollers inside the mixer chamber. Consequently, it was necessary to compensate to maintain the target of operational temperature of $120{ }^{\circ} \mathrm{C}$. Thereby, the set point temperature was reduced to $110^{\circ} \mathrm{C}$. The obvious reason for this is that at the elevated temperatures, crosslinking could be activated within mixing chamber; as a result, the viscosity of the material would increase and could cause damage to the equipment. Therefore, it was necessary to alter the chamber setup, i.e. temperature and torque to achieve the desired temperature profile. Fig. 3 shows the optimized response of torque at $30 \mathrm{RPM}$, a set point of $110^{\circ} \mathrm{C}$ and a mixing time of at least $15 \mathrm{~min}$.
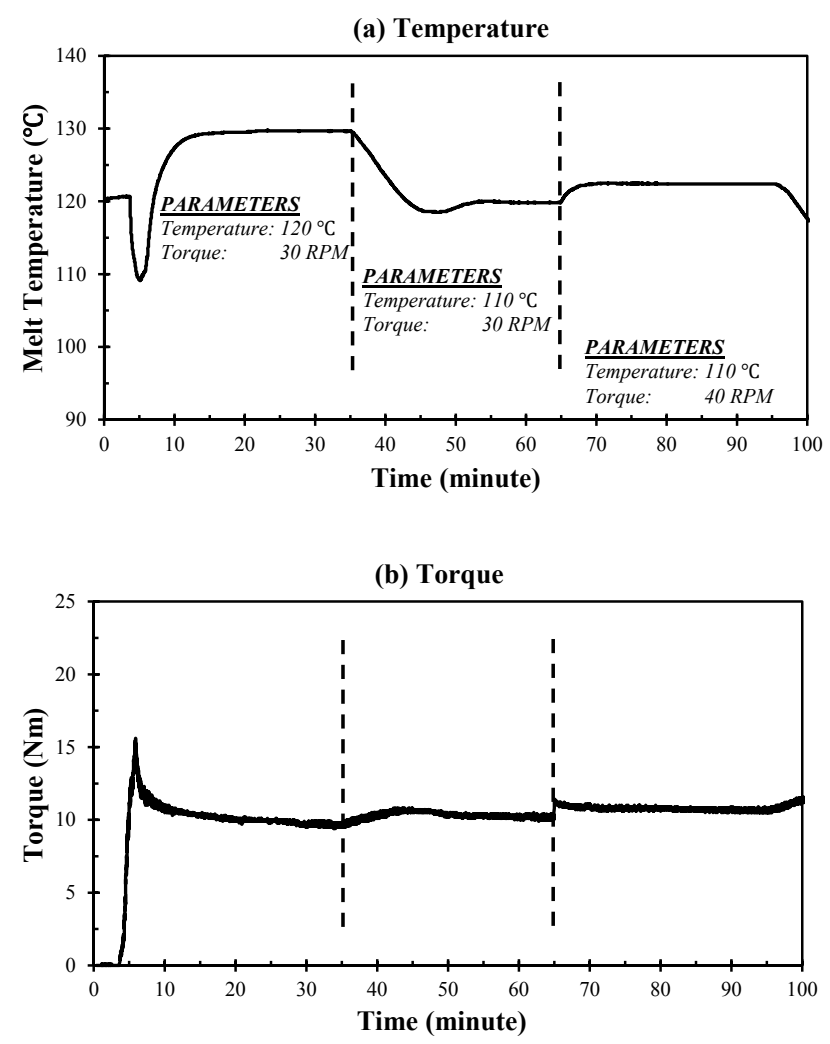

Fig. 2. Evaluation of temperature and torque conditions using the PolyLab mixer for LDPE: (a) melt temperatures (b) torque generated during the mixing process. 
(a) Temperature

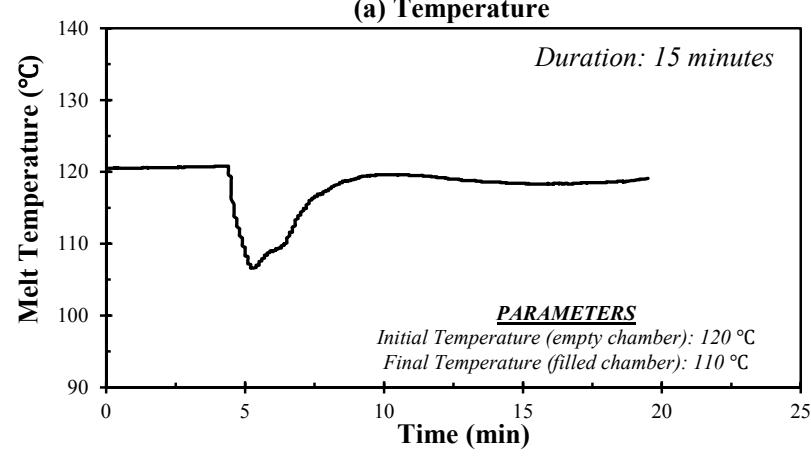

(b) Torque

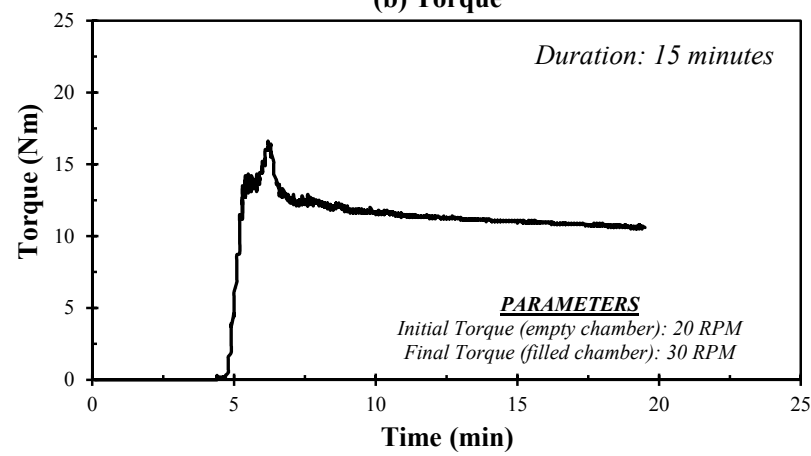

Fig. 3. Evaluation of temperature and torque conditions using the PolyLab mixer for LDPE grade material mixed with $2 \%$ DCP crosslinking agent: (a) melt temperature (b) the torque generated during the mixing process.

\section{B. Material Charactarzation}

To validate the uniformity of the crosslinking agent distribution within the prepared material, DSC analysis was performed and typical melting curves are shown in Fig. 4. The two peaks seen on heating (black trace) are associated with melting at $\sim 105^{\circ} \mathrm{C}$ and the crosslinking exothermic peak at $\sim 180-200{ }^{\circ} \mathrm{C}$. It can be seen from a second scan (Fig. 4, red trace), that the melting behavior is clearly affected by crosslinking. Similar effects were observed for all batches, confirming that crosslinking occurred during the first scan, as no sign of the exothermic peak (DCP decomposition) was observed in subsequent DSC scans. Moreover, analysis of exothermic area for all samples were undertaken and the resulting data were within the range of $\pm 1 \mathrm{~J} / \mathrm{g}$, indicating uniform DCP distribution within the material. In addition, this also supports the findings of Fig. 3, in which the parameters used for mixing purposes are optimized to achieve a uniform mixture.

It is well known that various factors could be responsible for affecting material properties during crosslinking. Besides external aspects, such as crosslinking temperature and time, a number of structural characteristics of the polymer strongly influence both the crosslinking ability and the resulting network. It is worth noting that crosslinking occurred within range of $160-220^{\circ} \mathrm{C}$ for LDPE system using DCP crosslinking agent, in line with results reported elsewhere [9]. These findings also validate the uniformity of the crosslink agent within the LDPE.

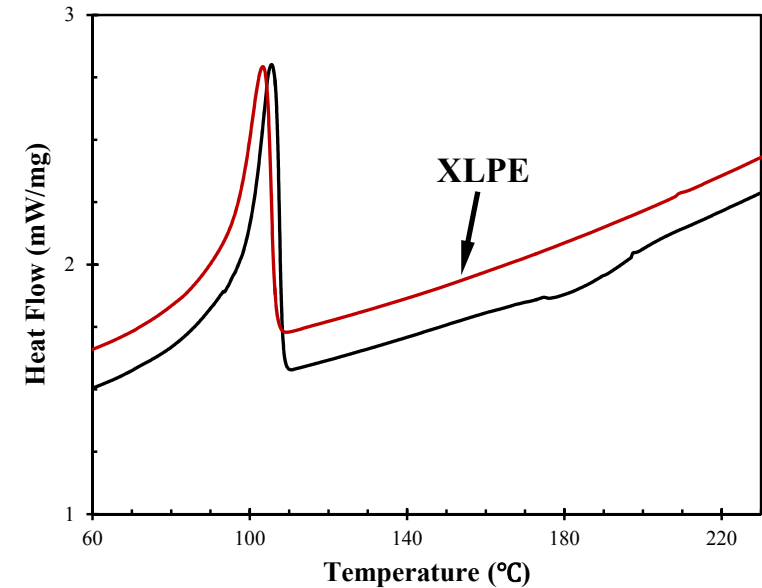

Fig. 4. DSC curve of Heat flow versus temperature for LDPE grade material mixed with $2 \%$ DCP. The samples are scanned at $10^{\circ} \mathrm{C} / \mathrm{min}$.

\section{XLPE Breakdown Strength}

Although, it is generally understood that electrical failure of a material occurs when a conductive pathway bridges the dielectric, the mechanisms that lead to this are complex [10, 11]. In this study, the DC breakdown strength of all three crosslinked LDPE grades (degassed and undegassed) was determined. The resulting data and Weibull parameters are presented in Table I. From Table I, it can be seen that material $C$ has the lowest breakdown strength suggesting a higher level of impurities, whilst $A$ and $B$ are the highest. The effects are more prominent in the degassed samples. Considering the uncertainties, it can be suggested that both materials $A$ and $B$ have comparable dielectric breakdown strength.

TABLE I. DC BREAKDOWN STRENGTH OF XLPE TESTED AT ROOM TEMPERATURE. NOTE, DATA SHOWS MEAN VALUES OF 20 TESTS PER BATCH.

\begin{tabular}{|c|c|c|c|}
\hline \multirow[b]{2}{*}{ Materials } & \multicolumn{3}{|c|}{ Weibull Results $(\mathrm{kV} / \mathrm{mm})$} \\
\hline & $\begin{array}{c}\text { Beta } \\
(\beta)\end{array}$ & $\begin{array}{c}E t a \\
(\eta)\end{array}$ & $\begin{array}{c}\text { Mean Breakdown Strength } \\
(\mathrm{kV} / \mathrm{mm} \pm \text { Std Dev })\end{array}$ \\
\hline \multicolumn{4}{|l|}{$A$ (reference) } \\
\hline Degassed & 21.55 & 501.72 & $489 \pm 29$ \\
\hline Un degassed & 17.13 & 440.68 & $427 \pm 34$ \\
\hline \multicolumn{4}{|l|}{$\boldsymbol{B}$} \\
\hline Degassed & 16.69 & 502.38 & $488 \pm 34$ \\
\hline Un degassed & 14.51 & 460.23 & $445 \pm 34$ \\
\hline \multicolumn{4}{|l|}{$C$} \\
\hline Degassed & 12.62 & 465.30 & $447 \pm 40$ \\
\hline Un degassed & 12.30 & 461.34 & $442 \pm 48$ \\
\hline
\end{tabular}

Breakdown in polymers is of great technological in connection with high voltage cable development. It is well known that the material structure and composition can influence dielectric strength [11] and, consequently, the purity of insulating systems is an important factor to consider in connection with enhanced material performance. Fig. 5 highlights the electrical strength (95\% confidence intervals) of both degassed and undegassed samples using Weibull distribution plots. It is worth noting, in all cases, degassed samples have the higher breakdown strength, indicating the 
positive influence of this process on the material. These findings clearly demonstrate that material purity and degassing can strongly influenced electrical breakdown strength.
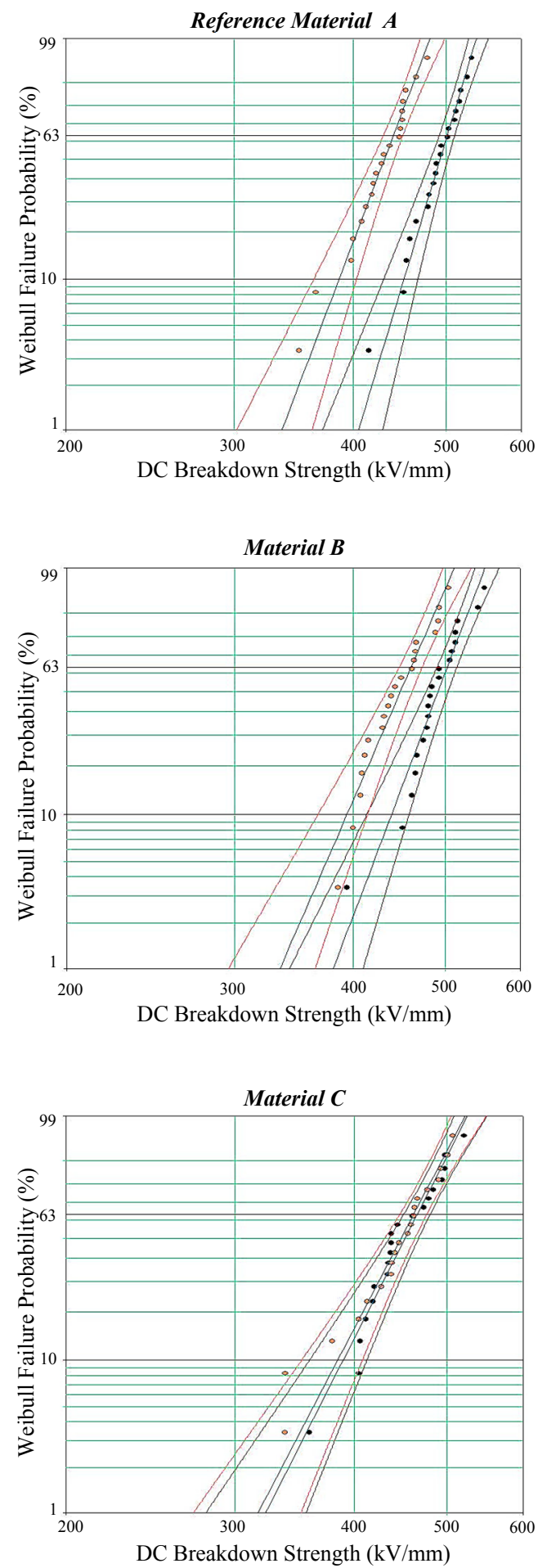

- Degassed • Undegassed

Fig. 5. Weibull probability plots of dielectric DC breakdown strength for XLPE samples measured at room temperature.

\section{CONCLUSIONS}

This study was undertaken in an attempt to explore the influence of processing parameters for various crosslinked LDPE materials on their breakdown behavior. Electrical tests on the resulting XLPE showed that materials $A$ and $B$ exhibit comparable breakdown strengths, while the performance of material $C$ has the lowest DC breakdown strength. Although, identical processing procedures were followed for all materials grades, the findings suggest that the differences in breakdown performance could be due to differences in material purity, that is, samples A and B being cleaner than C. Moreover, degassed XLPE samples (in all cases) have shown the highest breakdown strength, indicating a positive influence on the material performance of removal of crosslinking by-products. DSC plots revealed the radical crosslinking reaction occurs at $\sim 180{ }^{\circ} \mathrm{C}$ using DCP crosslink agent in a LDPE grade material. In addition, the current study also highlights processing conditions (parameters) required for uniform mixing of DCP within a polymeric host.

\section{ACKNOWLEDGMENT}

The authors are grateful for the financial support from the State Grid Cooperation of China: Research on Key Technologies of Insulation Material and Accessories for 320 kV HVDC XLPE Cable System (SGRIZLJS(2014)888).

\section{REFERENCES}

[1] B. Gustafsson, J.O. Bostrom, and R.C. Dammert, "Stabilization of peroxide crosslinked polyethylene," Die Angewandte Makromolekulare Chemie, vol. 261-262(1), pp. 93-99, 1998.

[2] B. Sultan, and M. Palmlof, "Advances in crosslinking technology," Plastics. rubber and composites. processing and applications, vol. 21, pp. 65-73, 1994.

[3] M. Lazar, M, R. Rado, and J. Rychly, "Crosslinking of polyolefins," in Polymer Physics, Springer, vol. 95, pp. 149-197, 1990.

[4] L. H. U. Andersson, and T. Hjertberg, "The effect of different structure parameters on the crosslinking behaviour and network performance of LDPE," Polymer, vol. 47, pp. 200-210, 2006.

[5] F. Gugumus, Plastics Additives, 3rd ed., Munich: Hanser, 1990.

[6] Y.L. Chong, G. Chen, H. Miyake, K. Matsui, Y. Tanaka, and T. Takada, "Space charge and charge trapping characteristics of cross-linked polyethylene subjected to ac electric stresses," J. Phys. D: Appl. Phys, vol. 39, pp. 1658-1666, 2006.

[7] H. Nuriziani, and C. George, "The trapping characteristic of low density polyethylene in the presence of crosslinking by-products," J. Phys: conference series, vol. 183, 2009.

[8] J. H. Mason, "Effects of thickness and area on the electric strength of polymers," IEEE Trans. Electri. Insul, vol. 26, pp. 318-322, 1991.

[9] F. Severini, and R. Gallo, "DSC study of thermal decomposition of peroxide and azo derivative mixtures in the presence of LDPE," Journal of thermal analysis, vol. 32, pp. 1189-1200, 1987.

[10] P. Fischer, "Dielectric Breakdown Phenomena in Polymers," in Electrical Properties of Polymers, D.A. Seanor, New York: Academic Press, 1982, pp. 319-364.

[11] C. C. Ku, and R. Leipens, Electrical Properties of Polymers, Munich: Hanser, 1987. 